\section{Jean-Yves Scoazec} Guy Lesèche

\section{ADRESSES}

J.-Y. Scoazec : maître de conférences des universités, praticien hospitalier. Laboratoire de biologie cellulaire et Inserm U. 327, faculté de médecine Xavier-Bichat, 16 , rue HenriHuchard, 75018 Paris, France.

G. Lesèche : praticien hospitalo-universitaire. Service de chirurgie thoracique et vasculaire, hôpital Beaujon, 92118 Clichy Cedex, France.

TIRÉS A PART

J.-Y. Scoazec.

\title{
Immunologie des cellules endothéliales et rejet de greffe
}

Les cellules endothéliales jouent un rôle de premier plan dans le rejet de greffe, à la fois comme actrices et comme cibles du rejet. En effet, elles sont les premières à interagir avec les cellules immunocompétentes du receveur et à recruter les lymphocytes et les macrophages qui vont relayer la réponse contre le greffon. Les cellules endothéliales sont également les premières cibles et victimes de cette réponse, humorale et cellulaire. La mise au point d'immunomodulateurs de la réponse immune aux cellules endothéliales du greffon, et de protecteurs efficaces de l'endothélium, constituent donc des recherches thérapeutiques importantes sur la tolérance aux greffes.

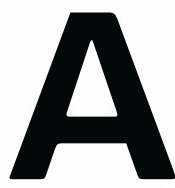
près une transplantation d'organe, les déterminants antigéniques associés aux antigènes du complexe majeur d'histocompatibilité $(\mathrm{CMH})$ du donneur stimulent les lymphocytes $\mathrm{T}$ du receveur et provoquent l'induction d'une réaction immunitaire qui peut aboutir au rejet de la greffe, c'est-à-dire à la destruction spécifique des cellules du donneur [1]. Au cours de la réaction de rejet, les cellules endothéliales du greffon jouent un rôle de premier plan, à la fois comme actrices et comme cibles. Actrices, car elles entretiennent des rapports fonctionnels privilégiés avec les leucocytes circulants, et donc, en situation de greffe, avec les cellules immunocompétentes du receveur. Cibles, car elles sont particulièrement exposées aux agressions des effecteurs humoraux et cellulaires de la réaction de rejet, non seulement en raison de leur position stratégique à l'interface entre le tissu greffé et le sang, mais aussi en raison de certaines de leurs propriétés intrinsèques. Ce sont ces deux aspects que nous envisagerons successivement.

\section{Les cellules endothéliales, actrices de la réaction de rejet}

L'une des propriétés essentielles des cellules endothéliales est leur capacité à établir des interactions fonctionnelles spécifiques et contrôlés avec les leucocytes circulants. Au cours de la transplantation et du rejet de greffe, cette propriété confère aux cellules endothéliales du greffon un rôle essentiel dans le recrutement des cellules immunocompétentes du receveur et contribue à leur donner un pouvoir immunogène. 
Les cellules endothéliales du greffon sont indispensables au recrutement des cellules immunocompétentes du receveur

La destruction spécifique des cellules du donneur, qui caractérise la réaction de rejet de greffe, nécessite le recrutement dans le greffon des cellules immunocompétentes du receveur. Ce recrutement comporte plusieurs étapes successives : (1) l'accumulation des cellules effectrices du receveur dans la microcirculation de l'organe greffé ; (2) l'adhérence de ces cellules à l'endothélium vasculaire ; et (3) leur migration à travers la paroi vasculaire vers le tissu sous-jacent.

L'accumulation des cellules effectrices du receveur dans le greffon est facilitée par l'effet chimiotactique de certaines cytokines, comme l'IL8 [2], et par les modifications des conditions rhéologiques locales. Ainsi, les cytokines IL1, tumor necrosis factor $\alpha$ (TNF $\alpha$ ), et peut-être IL2, modulent la production de médiateurs endothéliaux réglant le tonus vasculaire, et exercent ainsi un effet globalement vasodilatateur [2]. De plus, un effet rapide de l'IL1, du TNF $\alpha$ et de l'interféron $\gamma$ sur le cytosquelette endothélial conduit à une augmentation prolongée de la perméabilité du revêtement endothélial capillaire [3]. Ces modifications locales sont un préalable indispensable à l'établissement d'interactions stables entre les cellules endothéliales et les différentes populations de leucocytes circulants. La formation de ces interactions stables nécessite la présence de protéines d'adhérence spécialisées [4-8], dont les principales figurent dans le Tableau I. Deux d'entre elles, la Psélectine (ou PAGDEM) et la Esélectine (ou ELAM-1), appartiennent à la famille des sélectines. Les autres, ICAM-1, ICAM-2, VCAM-1 et LFA-3, appartiennent à la superfamille des immunoglobulines, qui doit son nom au fait que les protéines qui la composent ont un segment extracellulaire formé par plusieurs domaines de structure très caractéristique, identique à celle des domaines des chaînes d'immunoglobulines.

Une seule de ces protéines d'adhérence est exprimée à l'état normal sur l'ensemble des cellules endothéliales $\mathrm{m} / \mathrm{s} n^{\circ} 10$ vol. 9, octobre 93

\begin{tabular}{|c|c|c|c|}
\hline \multicolumn{3}{|c|}{ Tableau I } \\
PRINCIPALES PROTÉINES ENDOTHÉLIALES SPÉCIALISÉES \\
DANS L'ADHÉRENCE LEUCOCYTAIRE
\end{tabular}

capillaires. C'est ICAM-2, dont le ligand est une intégrine, LFA-1, exprimée sur l'ensemble des leucocytes circulants. Les autres protéines sont inductibles : elles ne sont normalement pas exprimées à la surface des cellules endothéliales capillaires (encore que de nombreuses exceptions à cette règle soient maintenant connues) et ne sont présentes que sur des cellules endothéliales stimulées par certains médiateurs de l'inflammation, comme l'IL1, l'interféron $\gamma$ et le TNF $\alpha[2,7]$. Chacune de ces protéines a un rôle précis au cours du processus d'adhérence leucocytaire, qui comprend deux phases principales (figure 1): (1) la première phase consiste dans le ralentissement du déplacement des leucocytes dans le flux circulatoire afin d'augmenter la fréquence et la durée de leurs contacts avec l'endothélium vasculaire ; (2) la seconde phase correspond à la stabilisation de l'adhérence entre les leucocytes et les cellules endothéliales. La première phase du processus est assurée par les sélectines (figure 1a). La P-sélectine, dont l'induction très rapide est provoquée, contrairement à celle des autres protéines d'adhérence, par des agents non spécifiques comme l'histamine, la thrombine et les prostaglandines, interagit préférentiellement avec les polynucléaires neutrophiles et les monocytes. La Esélectine, dont l'induction est plus tardive et dépend des médiateurs de l'inflammation, assure plus spécifiquement le recrutement des lymphocytes lors du déclenchement de la phase immune de la réaction inflammatoire.

La seconde phase du processus, celle de l'adhérence stable, fait intervenir notamment les protéines ICAM-1 et VCAM-1 dont les ligands leucocytaires appartiennent à la famille des intégrines (figure 1b). Les ligands de ICAM-1 sont les protéines LFA-1 et Mac1, présentes à la surface de l'ensemble des leucocytes circulants. Le ligand de VCAM-1 est VLA4, une intégrine caractéristique des lymphocytes T activés. En plus de ces deux couples principaux, d'autres protéines d'adhérence peuvent intervenir. Ainsi, la protéine LFA-3, dont l'un des facteurs inducteurs semble être l'IL4, est souvent détectée sur les endothéliums au voisinage des foyers inflammatoires : son ligand est CD2, une protéine membranaire présente sur l'ensemble des lymphocytes $\mathrm{T}$ circulants. 
1. Dausset $\mathrm{J}$. The major histocompatibility complex in man. Science 1981; 213: 1469-73.

2. Mantovani A, Dejana E. Cytokines as communication signals between leukocytes and endothelial cells. Immunol Today 1989 ; $10: 370-5$.

3. Martin S, Maruta K, Burkart V, Gillis $\mathrm{S}$, Kolb H. IL-1 and IFN- $\gamma$ increase vascular permeability. Immunology 1988 ; 64 : 301-5.

4. Springer TA. Adhesion receptors of the immune system. Nature $1990 ; 346$ : 425-34.

5. Springer TA, Lasky LA. Sticky sugars for selectins. Nature 1991; 349 : 196-7.

6. Shimizu Y, Newman W, Tanaka Y, Shaw S. Lymphocyte interactions with endothelial cells. Immunol Today 1992; 13 : 106-12.

7. Pober JS. Cytokine-mediated activation of vascular endothelium: physiology and pathology. Am J Pathol 1988 ; 133 : 426-33.

8. Fischer A, Auffray C, Durandy A. Les molécules d'adhérence des lymphocytes $T$. médecine/sciences $1987 ; 3: 334-42$.

9. Scoazec JY, Feldmann G. In situ immunophenotyping study of endothelial cells of the human hepatic sinusoid: results and functional implications. Hepatology 1991; 14: 789-97.

10. Adams DH, Hubscher SG, Shaw J, Rothlein R, Neuberger JM. Intercellular adhesion molecule 1 on liver allografts during rejection. Lancet 1989 ; 2 : 1122-5.

11. Steinhoff $\mathrm{G}$, Behrend $\mathrm{M}$, Wonigeit $\mathrm{K}$. Expression of adhesion molecules on lymphocytes/monocytes and hepatocytes in human liver grafts. Hum Immunol $1990 ; 28$ : 123-7.

12. Bishop GA, Hall BM. Expression of leucocyte and lymphocyte adhesion molecules in the human kidney. Kidney Int 1989 ; 36 : 1078-85.

13. Lafferty KJ, Prowse SJ, Silmeonovic CJ, Warren HS. Immunobiology of tissue transplantation : a return to the passenger leukocyte concept. Annu Rev Immunol 1983 ; $1: 143-58$

14. Rose ML, Page C, Hengstenberg C, Yacoub MH. Identification of antigen presenting cells in normal and transplanted human heart: importance of endothelial cells. Hum Immunol $1990 ; 28$ : 179-85.
15. Hirschberg $\mathrm{H}$, Braathen LR, Thorsby E. Antigen presentation by vascular endothelial cells and epidermal Langerhans cells : the role of HLA-DR. Immunol Rev 1982 ; 66 : $57-77$.

16. Pober JS, Collins T, Gimbrone MA Jr, Libby $\mathrm{P}$, Reiss CS. Inducible expression of class II major histocompatibility complex antigens and the immunogenicity of vascular endothelium. Transplantation 1986; 41 : 141-6.

17. Janeway C. Immunogenicity signals 1 , $2,3 \ldots$ and 0 . Immunol Today $1989 ; 10$ : 283-6.

18. Bouwman E, Ijzermans JNM, Heineman E, de Bruin RWF, Marquet RL, Jeekel J. Class II antigen expression on vascular endothelium of the graft in rat heart transplantation. Transplant Proc 1987 ; 19 : 198-9.

19. Milton AD, Spencer SC, Fabre JW Detailed analysis and demonstration of differences in the kinetics of induction of class I and class II major histocompatibility complex antigens in rejecting cardiac and kid ney allografts in the rat. Transplantation $1986 ; 41$ : 499-508.

20. Miossec P, Cavender D, Ziff M. Production of interleukin 1 by human endothelial cells. J Immunol 1986 ; 136 : 2486-91.

21. Ferry B, Halttunen J, Leszczynski D, Schellekens $\mathrm{H}$, van der Meide PH, Häyry $\mathrm{P}$. Impact of class II major histocompatibility complex antigen expression on the immunogenic potential of isolated rat vascular endothelial cells. Transplantation 1987 ; 44: 499-503.

22. Scoazec JY, Delautier D, Degott C Belghiti J, Feldmann G. Both endothelial and epithelial cells of UW-preserved human liver grafts express class II MHC molecules and leukocyte adhesion molecules. $J$ Hepatol 1992; 16 (suppl 1) : S43.

23. Platt JL, Lindman BJ, Geller RL, et al. The role of natural antibodies in the activation of xenogenic endothelial cells. Transplantation 1991; 52 : 1037-43.

24. Turman MA, Casali $P$, Notkins AL, Bach FH, Platt JL. Polyreactivity and antigen specificity of human xenoreactive monoclonal and serum natural antibodies. Transplantation $1991 ; 52$ : 710-7.

25. van den Bogaerde J, Aspinall R, Wang $\mathrm{MW}$, et al. Induction of long-term survival of hamster heart xenografts in rats. Transplantation $1991 ; 52$ : 15-20.
Quels sont le profil et la cinétique d'expression de ces protéines d'adhérence au cours de la transplantation et du rejet de greffe ? Malgré des informations encore fragmentaires, notamment aux phases précoces de la transplantation, on peut affirmer qu'il existe bien une induction coordonnée de l'expression des protéines d'adhérence spécialisées sur les cellules endothéliales des greffons. Nous prendrons deux exemples bien étudiés, celui des allogreffes hépatiques et celui des allogreffes rénales.

Dans le foie, les cellules endothéliales bordant le sinusoïde hépatique sont caractérisées par un niveau d'expression d'ICAM-1 constitutionnellement élevé [9]. En raison de cette particularité, les cellules endothéliales sinusoïdales des greffons hépatiques sont donc d'emblée des sites d'adhérence particulièrement favorables pour les lymphocytes du receveur. Au cours de la transplantation, comme le montrent les études immunohistochimiques, il existe une induction d'ICAM-1, de ELAM-1 et de LFA-3 sur les sinusoïdes et les petits capillaires des espaces portes des greffons hépatiques $[10,11]$. L'induction de ces protéines ne semble pas corrélée avec la survenue d'une réaction de rejet : elle ne fait que traduire indirectement le développement de la réponse immunitaire du receveur.

Dans le rein, la situation est plus complexe. A l'état normal, les capillaires péritubulaires expriment des niveaux significatifs d'ICAM-1 et de LFA-3 [12]. Ces protéines seraient peu induites au cours de la transplantation. Leur expression pourrait même artificiellement diminuer au cours du rejet, en raison de la destruction des capillaires qui les expriment. Il n'existe pas d'information précise sur le comportement des autres protéines d'adhérence endothéliales au cours de la transplantation rénale. En revanche, le rejet des allogreffes rénales semble s'accompagner de l'induction de l'expression de l'intégrine LFA-1 sur les capillaires péritubulaires, ce qui permettrait l'adhérence de leucocytes exprimant ICAM-1, dont les monocytes et les lymphocytes activés.

$\mathrm{m} / \mathrm{s} n^{\circ} 10$ vol. 9 , octobre 93 


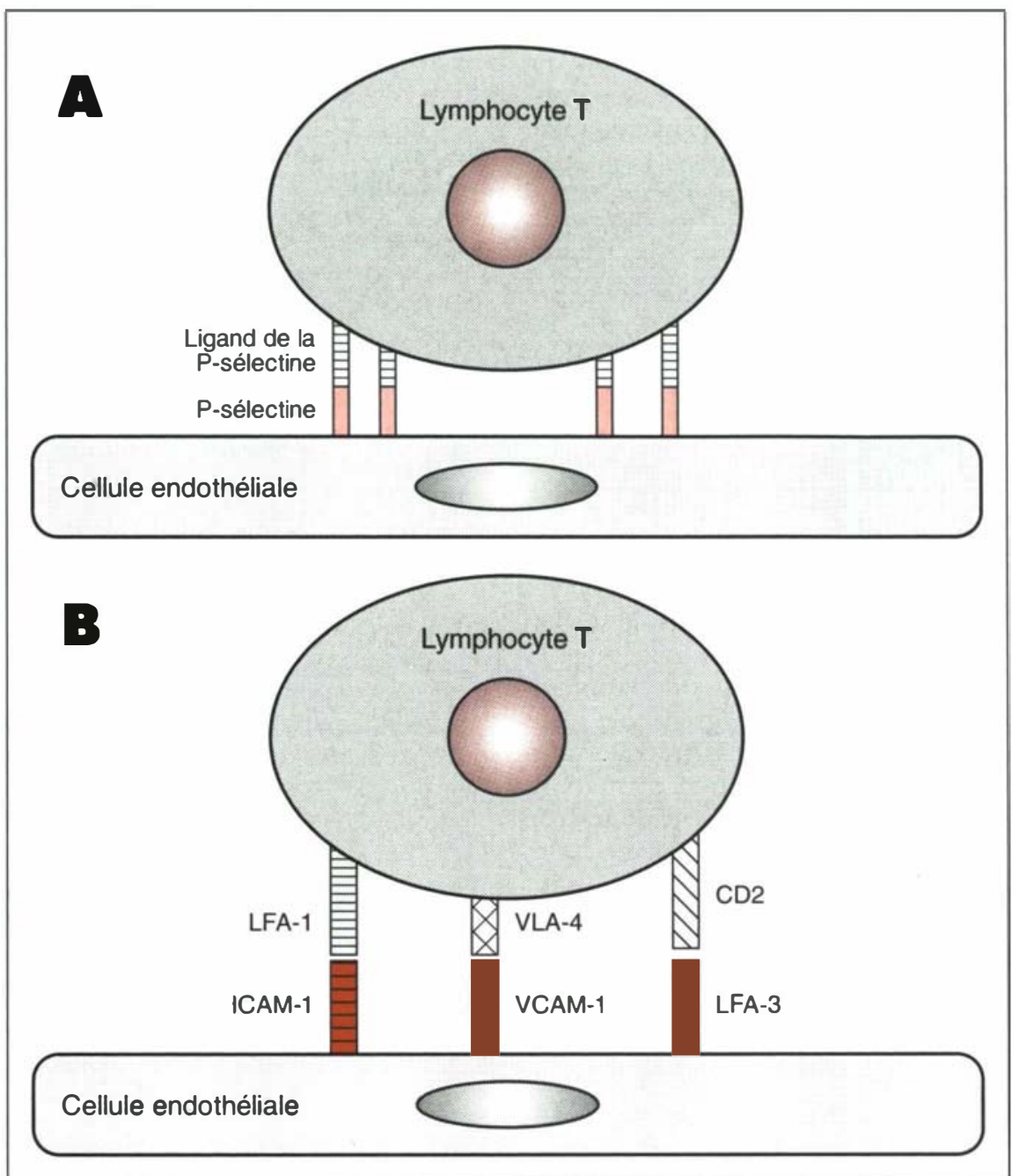

Figure 1. Mécanismes moléculaires impliqués. (A) dans le ralentissement des lymphocytes. (B) dans leur adhérence à l'endothélium vasculaire stimulé. ICAM-1 : intercellular adhesion molecule-1 ; VCAM-1 : vascular cell adhesion molecule-1 ; LFA-3 : lymphocyte function antigen-3; VLA : very late antigen.

Les cellules endothéliales jouentelles un rôle dans la présentation des allo-antigènes?

L'immunogénicité d'un greffon a été longtemps expliquée par l'hypothèse dite du "leucocyte voyageur ", selon laquelle un greffon n'est immunogène que s'il contient encore des cellules spécialisées capables de présenter aux lymphocytes $\mathrm{T}$ du receveur les alloantigènes du donneur associés au $\mathrm{CMH}$ [13]. Ces cellules spécialisées dans la présentation de l'antigène peuvent être des monocytes circulants piégés dans la microcirculation du greffon au moment de son prélèvement ou les macrophages tissulaires et les cellules dendritiques normalement résidant dans le greffon.

$\mathrm{m} / \mathrm{s} n^{\circ} 10$ vol. 9, octobre 93 non sensibilisés préalablement [16]. In vivo, pour qu'une cellule présente efficacement des antigènes, elle doit remplir au moins trois conditions [17] : (1) elle doit exprimer à sa surface les molécules de classe II du $\mathrm{CMH}$, puisque les lymphocytes $\mathrm{T}$ auxiliaires ne reconnaissent l'antigène que si celui-ci, préalablement modifié et dégradé sous forme de petits peptides, est exposé à la surface de la cellule en association avec ces molécules ; (2) elle doit exprimer des protéines d'adhérence spécialisées capables d'interagir avec les protéines correspondantes exprimées par les lymphocytes $\mathrm{T}$ auxiliaires; ces couples de protéines d'adhérence, dont les plus importants sont ICAM-1/LFA-1 et LFA-3/CD2, assurent des interactions cellulaires non spécifiques stabilisant et renforçant la liaison spécifique entre l'antigène et son récepteur lymphocytaire (figure 2) ; (3) enfin, elle doit être capable de sécréter des médiateurs solubles stimulant la prolifération des lymphocytes $\mathrm{T}$; les principaux de ces médiateurs sont l'IL1 et l'IL6 [6] (figure 3).

Les cellules endothéliales des tissus greffés remplissent au moins une partie de ces conditions. En effet, il a été vérifié, notamment par immunohistochimie, qu'elles expriment de façon quasi constante les molécules de classe II du $\mathrm{CMH}$, quel que soit le niveau d'expression de ces antigènes dans le tissu normal [18, 19]. De plus, comme nous l'avons vu, les cellules endothéliales capillaires des tissus greffés expriment les protéines d'adhérence spécialisées, notamment ICAM-1 et LFA-3, nécessaires aux interactions avec les lymphocytes $\mathrm{T}$ auxiliaires. Leur capacité de production d'IL1 et 6 n'a pas été étudiée directement. Elle est toutefois possible, puisque la production d'IL6 est considérée comme une propriété constitutionnelle des cellules endothéliales [2], et que la synthèse d'IL1 peut être induite par les cytokines inflammatoires [20]. On peut donc admettre que les cellules endothéliales des tissus greffés sont susceptibles de jouer un rôle dans la présentation des alloantigènes. Des arguments directs en ce sens ont d'ailleurs été obtenus dans des modèles expérimentaux de transplantation cardiaque chez le rat [21]. 


\section{RÉFÉRENCES}

26. Kowalski L, Falqui L, Lacy PE, Scharp DW. Production of marked prolongation of survival of canine islet xenografts in mice by antilymphocyte sera and L3T4 antibody. Transplantation 1991; 52 : 1094-7.

27. Adorini L, Muller S, Cardinaux F, Lehmann PV, Falcioni $F$, Nagy $Z A$. In vivo competition between self peptides and foreign antigens in T-cell activation. Nature 1988 ; $334: 623-5$.

28. Faustman D, Coc C. Prevention of xenograft rejection by masking donor HLA class I antigens. Science $1991 ; 252: 1700-2$.

29. Dalmasso AP, Vercelotti GM, Platt JL, Bach FH. Inhibition of complementmediated endothelial cell cytotoxicity by decay accelerating factor. Transplantation $1991 ; 52: 530-3$.

30. Bishop GA, Vaugh JA, Landers DV, Krensky AM, Hall BM. Microvascular destruction in renal transplant rejection. Transplantation 1989 ; 48 : 408-14.

31. Paul LC, van Es LA, van Rood JJ, van Leeuwen A, Brutel de la Rivière $G$, de Graeff J. Antibodies directed against antigens on the endothelium of peritubular capillaries in patients with rejecting renal allografts. Transplantation 1979 ; 27 : 175-9.

32. Dunn MJ, Rose ML, Latif $\mathrm{N}$, et al. Demonstration by Western blotting of antiheart antibodies before and after cardiac transplantation. Transplantation 1991; 51 : 806-12.

33. Paul LC, Baldwin III WM, van Es LA. Vascular endothelial alloantigens in renal transplantation. Transplantation $1985 ; 40$ : 117-23.

34. Rose EA, Smith CR, Petrossian GA, Barr ML, Reemstma K. Humoral immune responses after cardiac transplantation : correlation with fatal rejection and graft atherosclerosis. Surgery $1989 ; 106$ : 203-8.

35. Downey III WE, Baldwin III WM, Sanfilipo F. Association of donor-specific blood transfusion enhancement of rat renal allografts with accelerated development of antiidiotypic antibodies and reduced alloantibody responses. Transplantation $1990 ; 49$ : $160-6$.

36. Bender JR, Pardi R, Kosek J, Engleman EG. Evidence that cytotoxic lymphocytes alter and traverse allogeneic endothelial cell monolayers. Transplantation $1989 ; 47$ :
Un dernier point reste cependant à résoudre. Compte tenu des forces qui s'exercent sur les parois vasculaires, les lymphocytes $T$ ne peuvent adhérer de façon prolongée à l'endothélium que si le flux sanguin est ralenti et/ou si les cellules endothéliales expriment à leur surface des protéines d'adhérence spécialisées, et notamment, les protéines de la famille des sélectines [4]. Dans quelles conditions et à quel moment les conditions rhéologiques sont-elles favorables pour que les cellules endothéliales stimulées du donneur puissent interagir efficacement avec les lymphocytes $\mathrm{T}$ du receveur ? Jusqu'à présent, sur la base des modèles expérimentaux de transplantation chez l'animal, il est admis que les caractères phénotypiques de la microcirculation du tissu greffé ne sont modifiés significativement que plusieurs jours après la transplantation. Ces modifications seraient donc trop tardives pour permettre aux cellules endothéliales de jouer un rôle dans l'induction de la réponse immunitaire contre le greffon. Elles n'interviendraient que dans l'entretien de cette réponse immunitaire. Toutefois, des résultats récents obtenus dans notre laboratoire montrent que l'induction des molécules de classe II du $\mathrm{CMH}$ et des protéines d'adhérence spécialisées est probablement plus précoce qu'il n'est généralement admis. Dans des greffons hépatiques cryopréservés, nous avons pu, en effet, observer, avant même la réimplantation, une induction des antigènes de classe II du $\mathrm{CMH}$ et des protéines d'adhérence spécialisées sur les cellules endothéliales sinusoïdales [22]. Cette expression, même transitoire, pourrait permettre aux cellules endothéliales de jouer un rôle dans l'induction de la réponse immunitaire spécifique.

\section{Les cellules endothéliales, cibles de la réaction de rejet}

De nombreux arguments morphologiques et expérimentaux montrent que les cellules endothéliales sont les principales cibles de la réaction de rejet, qu'il s'agisse de rejet hyperaigu, aigu ou chronique. Ces lésions vasculaires sont essentiellement d'origine immunologique. Elles sont dues à l'action d'anticorps circulants et aux effets cytotoxiques des cellules immunitaires du receveur.

\section{Cellules endothéliales et rejet hyperaigu}

Les réactions de rejet hyperaigu sont essentiellement observées en cas de xénogreffe, c'est-à-dire de greffe entre espèces différentes, ou d'allogreffes entre sujets $\mathrm{ABO}$ incompatibles. La principale cible du rejet hyperaigu est la cellule endothéliale vasculaire qui est détruite, en présence du complément, par des anticorps anti-cellules endothéliales. Ces anticorps sont le plus souvent naturels et souvent polyréactifs, c'est-à-dire capables de réagir avec plusieurs molécules différentes, présentant des déterminants antigéniques communs [23, 24]. Les lésions sont précoces, sévères et étendues ; elles se traduisent en particulier par des thromboses massives du greffon. Les lésions du rejet hyperaigu ne sont pas contrôlées par les thérapeutiques anti-rejet habituelles, qui sont essentiellement actives sur l'immunité cellulaire. Seule la déplétion du sang circulant en anticorps ou en complément s'est montrée capable d'augmenter la durée de survie des xénogreffes dans des modèles animaux expérimentaux [25]. Deux stratégies thérapeutiques expérimentales ont été particulièrement explorées. La première consiste à bloquer les interactions entre les lymphocytes $\mathrm{T}$ cytotoxiques du receveur et les cellules greffées. Ce blocage peut être obtenu en interférant avec les molécules d'adhérence exprimées par les lymphocytes $\mathrm{T}$ eux-mêmes, telles que le récepteur de l'antigène, la molécule CD4 ou les molécules de classe II du $\mathrm{CMH}[26,27]$. Toutefois, cette stratégie nécessite un traitement systémique du receveur qui interfère avec ses capacités de réponse immunitaire. Une stratégie alternative est de bloquer les sites d'adhérence exprimés par les cellules greffées, telles que les molécules de classe I du $\mathrm{CMH}$, avant leur transplantation; cette technique, sans conséquence sur le receveur, a été notamment testée dans un modèle de xénogreffe pancréatique [28]. Une autre stratégie thérapeutique, récemment explorée, est la protection des cellules endothéliales contre l'action du complément. Ainsi, l'expression 


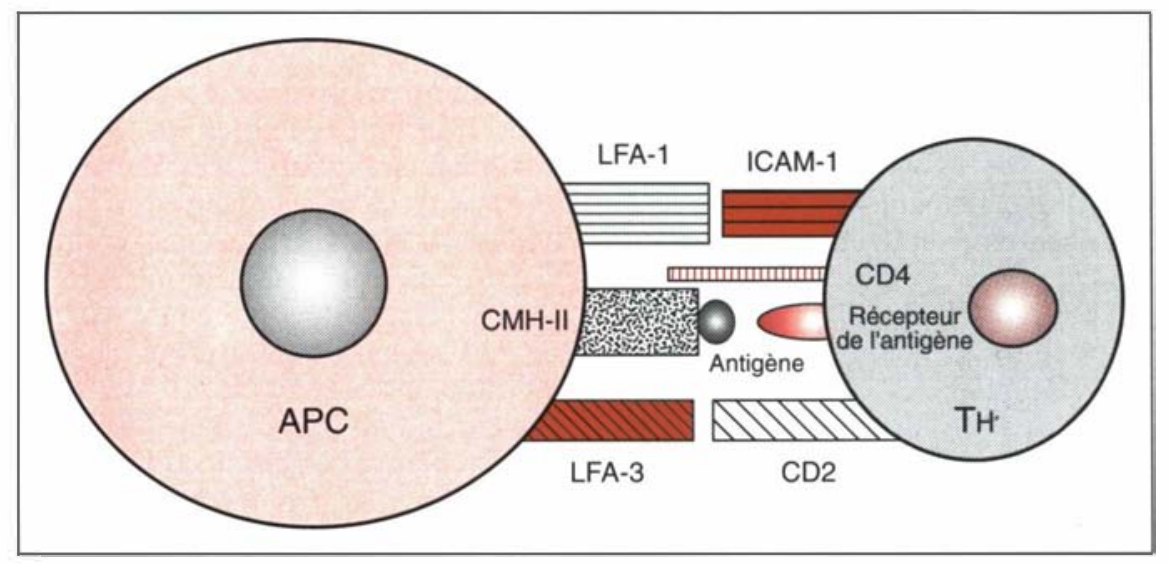

Figure 2. Interactions moléculaires entre une cellule spécialisée dans la présentation de l'antigène (APC) et un Iymphocyte T facilitant (TH) lors de la reconnaissance d'un antigène. CMH-II : molécule de classe II du complexe majeur d'histocompatibilité.

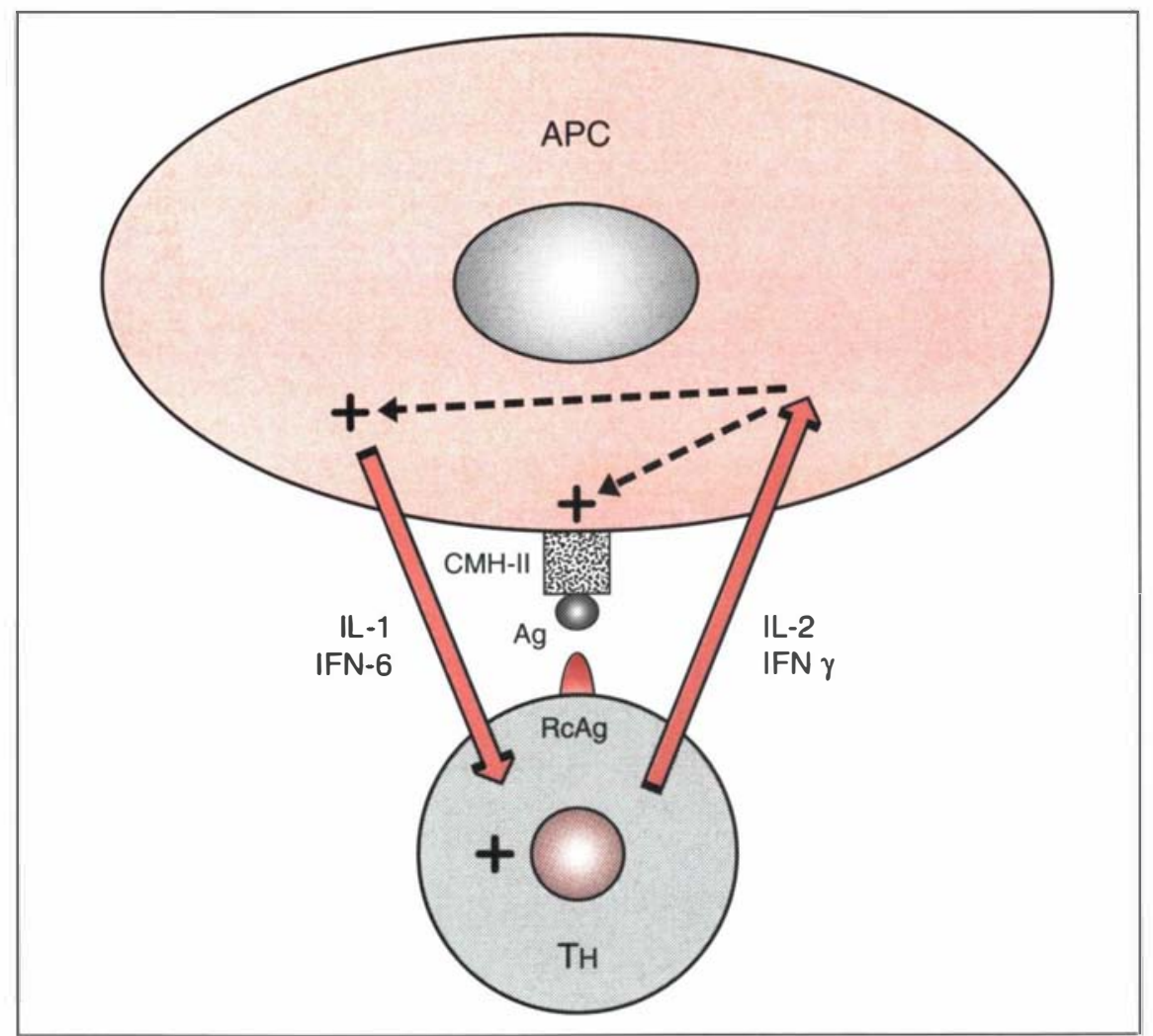

Figure 3. Interactions fonctionnelles entre une cellule présentatrice d'antigène (APC) et un lymphocyte $T$ facilitant (TH). Ag, antigène ; RcAg: récepteur de l'antigène; IL 1, interleukine 1; IL2, interleukine $2 ; I L 6$; interleukine 6 : IFN $\gamma$ : interféron $\gamma$. RcAg: récepteur de l'antigène. dans des cellules endothéliales d'une protéine inhibitrice du complément, le decay accelerating factor, d'origine érythrocytaire, a permis de protéger ces cellules de l'action cytotoxique, dépendante du complément, d'un sérum hétérologue [29]. La transposition de ces techniques en situation clinique sera sans doute délicate. Il s'agit néanmoins d'une direction de recherche importante car les xénogreffes pourraient constituer une alternative à la pénurie actuelle en allogreffes.

\section{Cellules endothéliales et rejet aigu}

Au cours du rejet aigu, ce sont les capillaires qui constituent l'une des principales cibles de la réaction immunitaire [30]. Le fait a été bien démontré dans le rein, où ce sont les capillaires péritubulaires qui présentent les lésions les plus précoces et les plus sévères, et dans le cœur, où les capillaires myocardiques sont les plus altérés. Dans les greffons hépatiques, il existe également des signes de souffrance endothéliale au cours des réactions de rejet, mais ces lésions sont plus difficiles à interpréter en raison de l'importance des lésions endothéliales induites par la conservation même des greffons.

Les lésions endothéliales du rejet aigu sont provoquées par des mécanismes humoraux et cellulaires. Des anticorps spécifiques dirigés contre des constituants de la membrane plasmique des cellules endothéliales ont été mis en évidence chez des receveurs d'allogreffes rénales [31] et cardiaques [32]. Certains de ces anticorps sont naturels. D'autres sont acquis à la suite de la réaction immunitaire induite par la transplantation. Les cibles de ces anticorps sont souvent les antigènes de classe II ou de classe I du CMH. Dans d'autres cas, les antigènes reconnus sont des antigènes indépendants du $\mathrm{CMH}$, notamment des antigènes communs aux cellules endothéliales et aux monocytes [33] et qui peuvent donc être détectés par la technique du crossmatch monocytaire afin de permettre une meilleure sélection des receveurs [34]. Rappelons que l'une des hypothèses expliquant le rôle protecteur des transfusions de sang effectuées chez le receveur préalablement à la transplantation, notamment dans le cas d'allogreffe rénale, est l'induc- 


\section{RÉFÉRENCES}

37. Kirby JA, Givan AL, Shenton BK, et al. Renal allograft rejection. Possible involvement of antibody-dependent cell-mediated cytotoxicity. Transplantation 1990 ; 50 : 225-9.

38. Suitters A, Rose M, Higgins A, Yacoub MH. MHC antigen expression in sequential biopsies from cardiac transplant patients - correlation with rejection. Clin Exp Immunol $1987 ; 69$ : 575-83.

39. Tilney NL, Whitley WD, Diamond JR, Kupiec-Weglinski JW, Adams DH. Chronic rejection - an undefined conundrum. Transplantation $1991 ; 52: 389-98$.

40. Suciu-Foca N, Reed E, D'Agati VD, et al. Soluble HLA antigens, anti-HLA antibodies, and antiidiotypic antibodies in the circulation of renal transplant recipients. Transplantation $1991 ; 51: 593-601$.

41. Forbes RDC, Gomersall M, Darden AG, Guttmann RD. Multiple patterns of MHC class II antigen expression on cellular constituents of rat heart grafts. Lack of correlation with survival, but strong correlation with vasculitis. Transplantation 1991 ; 51: 942-8.

42. Hruban RH, Beschorner WE, Baumgartner WA, et al. Accelerated arteriosclerosis in heart transplant recipients is associated with a T-lymphocyte-mediated endothelialitis. Am J Pathol $1990 ; 137$ : 871-82

43. Schmitz-Rixen T, Megerman J, Colvin RB, Williams AM, Abbott WM. Immunosuppressive treatment of aortic allografts. $J$ Vasc Surg 1988; 7 : 82-92.

44. Baumann G, Borel JC. Mćcanismes moléculaires de l'action des agents immunosuppresseurs. médecine/sciences $1992 ; 8$ : 366-71

45. Yamanoi $A$, Nagasue $N$, Kohno $H$, Chang YC, Hayashi T, Nakamura T. Attenuation of ischemia-reperfusion injury of the liver in dogs with cyclosporine. Transplantation $1991 ; 52: 27-30$.

46. Marin ML, Hardy MA, Gordon RE, Reemtsma K, Benvenisty AI. Immunomodulation of vascular endothelium : effects of ultraviolet B irradiation on vein allograft rejection. J Vasc Surg 1990 ; 11: 103-11.

47. Isobe $M$, Yagita $H$, Okumura $K$, Ihara A. Specific acceptance of cardiac allograft after treatment with antibodies to ICAM-1 and LFA-1. Science 1992; $255: 1125-7$. tion de la synthèse d'anticorps antiidiotypiques neutralisant ces anticorps cytotoxiques [35].

En dehors des anticorps circulants, plusieurs types de cellules immunitaires, dont les lymphocytes $\mathrm{T}$ cytotoxiques et les cellules NK stimulées par les lymphokines, sont capables de provoquer la lyse de cellules endothéliales. Le processus a été bien étudié expérimentalement [36], et dépend de la présence des antigènes de classe I du $\mathrm{CMH}$, qui jouent vis-à-vis des lymphocytes $\mathrm{T}$ cytotoxiques le même rôle que les antigènes de classe II visà-vis des lymphocytes $\mathrm{T}$ auxiliaires, et des protéines d'adhérence dépendantes des cytokines, dont ICAM-1 et LFA-3. Au sein d'un organe greffé, toutes les conditions sont réunies pour que les cellules endothéliales soient la cible des lymphocytes cytotoxiques [37]. L'expression des antigènes de classe I du CMH, présents à l'état normal sur les cellules endothéliales capillaires, est très stimulée par les cytokines inflammatoires, notamment par l'interféron $\gamma$. Certaines études suggèrent d'ailleurs que l'intensité des lésions de rejet est micux corrélée au niveau d'expression des antigènes de classe I du CMH qu'à celui des antigènes de classe II [38]. De plus, les cellules endothéliales capillaires du tissu greffé expriment habituellement, comme nous l'avons déjà décrit, les protéines d'adhérence dépendantes des cytokines. Nous avions vu l'importance de ce phénomène pour le recrutement des cellules immunocompétentes du receveur. Son corollaire inévitable est de faire des cellules endothéliales des cibles privilégiées pour les cellules effectrices du receveur.

\section{Cellules endothéliales et rejet chronique}

Le rejet chronique est défini par la persistance d'une réaction immunitaire chez le receveur plusieurs mois ou plusieurs années après la greffe, malgré la poursuite du traitement immunosuppresseur. Les lésions vasculaires sont souvent au premier plan des lésions de rejet chronique [39]. Il s'agit essentiellement de lésions artérielles, contrairement aux lésions du rejet aigu, qui touchent surtout les capillaires. Dans les allogreffes rénales, les lésions les plus évocatrices du rejet chronique sont des lésions d'artérite fibro-oblitérante et de fibrose glomérulaire. Dans les greffes cardiaques en rejet chronique, les lésions caractéristiques sont des lésions d'artériosclérose coronaire. A la différence des lésions d'artériosclérose coronaire spontanée, ces lésions sont concentriques et touchent habituellement toute la longueur des vaisseaux ; elles s'accompagnent d'un infiltrat inflammatoire riche en lymphocytes $\mathrm{T}$. Des lésions identiques peuvent se développer dans les artères des greffons hépatiques, dans les artérioles et les veines des greffons pulmonaires.

Le développement des lésions vasculaires du rejet chronique est probablement expliqué par la survenue d'épisodes répétés d'agression endothéliale, entraînant une prolifération intimale secondaire et une oblitération progressive de la lumière vasculaire. La lésion endothéliale initiale est sans doute due à des anticorps cytotoxiques circulants, agissant en présence du complément [39, 40]. Les lésions initiales sont ensuite entretenues et progressivement aggravées par l'accumulation locale de macrophages, de lymphocytes T cytotoxiques et de cellules NK [39]. Il est difficile de déterminer si l'accumulation locale de ces cellules traduit un phénomène non spécifique, de nature inflammatoire, ou si elle représente une réaction immunitaire spécifique dirigée contre les allo-antigènes encore exprimés par les cellules vasculaires du greffon [41, 42]. A cet égard, il est intéressant de noter que, bien que l'expression des antigènes de classe II du CMH soit inconstante, son niveau semble corrélé à la survenue des lésions vasculaires.

\section{Cellules endothéliales et tolérance immunitaire}

La production d'anticorps ou de cellules spécifiques des alloantigènes du donneur n'a pas constamment des effets négatifs sur l'évolution du greffon. Elle peut au contraire avoir des conséquences bénéfiques en induisant un état de tolérance. Ainsi, le traitement du receveur par des anticorps dirigés contre les alloantigènes du donneur peut, en apparence paradoxalement, induire une facilitation passive favorisant la tolérance de la greffe. De même, la pré-immunisation active du receveur avec des cellules allogéniques du donneur est capable d'induire un état de tolérance immunitaire. Les 
causes de cette tolérance sont multiples et ne sont encore que partiellement connues. Le masquage d'alloantigènes du donneur par des anticorps du receveur, la suppression des cellules $\mathrm{T}$ auxiliaires du receveur ou l'induction de cellules $\mathrm{T}$ suppressives spécifiques des alloantigènes du donneur contribuent probablement à la tolérance immédiate et à long terme des allogreffes. Le rôle des cellules endothéliales dans le développement des mécanismes de tolérance n'a été que peu ou pas exploré. Plusieurs questions seraient intéressantes à résoudre. Comment le blocage par des anticorps du receveur de certains alloantigènes exposés par les cellules endothéliales peut-il contribuer à la facilitation immunitaire ? Les cellules endothéliales interagissent-elles avec les lymphocytes $\mathrm{T}$ suppresseurs impliqués dans la tolérance de la greffe de la même façon qu'avec les lymphocytes $\mathrm{T}$ cytotoxiques? Une meilleure compréhension du rôle des cellules endothéliales dans les mécanismes de tolérance immunitaire pourrait orienter vers de nouvelles stratégies thérapeutiques, s'ajoutant à celles qui ont déjà été proposées pour induire une immunomodulation de l'endothélium et que nous allons maintenant présenter.

\section{Modifications thérapeutiques des propriétés immunologiques des cellules endothéliales}

Il est tentant de chercher à agir sur les cellules endothéliales des greffons pour diminuer leur pouvoir immunogène et/ou pour les protéger des lésions d'origine immunologique. Plusieurs types de traitements ont été testés : (a) les traitements immunosuppresseurs classiques; (b) des traitements spécifiques aux cellules endothéliales, souvent encore expérimentaux; et (c) l'utilisation d'anticorps antiprotéines d'adhérence.

Les agents immunosuppresseurs conventionnels, et notamment la ciclosporine, exercent des effets protecteurs sur les cellules endothéliales. La mise en évidence de ces effets est particulièrement facile dans les allogreffes vasculaires, au cours desquelles l'administration de ciclosporine à doses efficaces réduit l'intensité des lésions des cel-

$m / s n^{\circ} 10$ vol. 9, octobre 93 lules endothéliales et musculaires lisses et diminue l'infiltration leucocytaire [43]. Le mécanisme d'action de la ciclosporine est évidemment multifactoriel [44]. L'inhibition de la prolifération des lymphocytes $\mathrm{T}$ et de la synthèse des cytokines induite par la ciclosporine réduit l'induction des molécules de classe II et des protéines d'adhérence spécialisées sur les cellules endothéliales du greffon. En conséquence, les cellules endothéliales voient diminuer leur pouvoir immunogène et leurs capacités de provoquer l'adhérence des lymphocytes du receveur. Il n'est pas exclu que la ciclosporine puisse également exercer des effets directs sur les cellules endothéliales, notamment des effets stabilisants sur certains organites cellulaires permettant de réduire les lésions non spécifiques d'origine ischémique. Ainsi, dans le modèle de la transplantation hépatique chez le chien, le prétraitement des donneurs par la ciclosporine réduit significativement les lésions d'ischémie observées dans les foies prélevés et améliore la survie de l'organe lorsqu'il est greffé [45].

Certaines techniques spécifiquement conçues pour entraîner une immunomodulation de l'endothélium ont été testées expérimentalement. L'une de ces techniques est l'irradiation des allogreffes par les ultraviolets B [46], qui s'est montrée capable d'abolir la réaction immunitaire contre les antigènes du donneur dans plusieurs modèles d'allogreffes et de xénogreffes. Une des conséquences principales de l'irradiation par les ultraviolets B est de diminuer l'induction des molécules de classe II du $\mathrm{CMH}$ et des protéines d'adhérence endothéliales, dont ICAM-1.

Enfin, plusieurs équipes ont montré que des anticorps bloquant les sites actifs de protéines d'adhérence impliquées dans les interactions cellulaires entre cellules endothéliales et leucocytes, dont ICAM-1 ou son ligand LFA-1 [47], sont capables d'allonger la durée de survie des allogreffes tissulaires, de diminuer l'intensité des lésions vasculaires en cas de réaction de rejet, et de contribuer à contrôler des épisodes de rejet aigu. Il s'agit là d'une voie thérapeutique prometteuse.

En conclusion, les cellules endothéliales occupent une position clé au cours de la réaction de rejet de greffe, non seulement en raison de leur situation à l'interface entre le sang et les leucocytes, mais aussi en raison de leurs propriétés fonctionnelles, qui leur permettent de participer directement au recrutement et sans doute à la stimulation des cellules immunocompétentes du receveur. Les propriétés des cellules endothéliales sont cependant à double tranchant: leur situation les expose aux effets des anticorps spécifiques, naturels ou acquis, présents chez le receveur et leurs caractéristiques fonctionnelles en font des cibles privilégiées pour les cellules cytotoxiques. L'une des directions de recherche pour améliorer la survie et la tolérance des greffons est donc la mise au point de traitements immunomodulateurs et protecteurs de l'endothélium

\section{Summary}

Endothelial cells immunology and graft rejection

Endothelial cells play a critical role in the induction of graft rejection. Endothelial cell adhesion molecules, specialized in the interactions with leukocytes, are essential for the recruitment of recipient immunocompetent cells inside allografts. Furthermore, endothelial cells, stimulated by the cytokines secreted by recipient immune cells, might behave as antigen-presenting cells, contributing to the maintenance of the immune reaction against the graft. Endothelial cells are also targets of the graft rejection, as shown by the importance of vascular lesions in all forms of rejection, hyperacute, acute or chronic. Endothelial lesions are immunologically induced by both humoral and cellular mechanisms. Circulating anti-endothelial antibodies, most of ten natural, might induce, in a complement dependent fashion, endothelial cell lysis. Stimulated cytotoxic $\mathrm{T}$ lymphocytes and NK cells are also able to provoke endothelial alterations in grafted tissue. Immunomodulation and protection of the endothelium are therefore an important aim to achieve for the improvement of graft survival and tolerance. 